\title{
Ethical Issues in Genome Editing using Crispr/Cas9 System
}

\section{Rodriguez E}

Interdisciplinary Center for Studies on Bioethics, University of Chile, Chile

"Corresponding author: Rodriguez E, Interdisciplinary Center for Studies on Bioethics, University of Chile, Chile, Tel: 56-2-26031223; E-mail: erodriguezchi@gmail.com Received date: March 03, 2016; Accepted date: March 22, 2016; Published date: March 24, 2016

Copyright: $\odot 2016$ Rodriguez E. This is an open-access article distributed under the terms of the Creative Commons Attribution License, which permits unrestricted use, distribution, and reproduction in any medium, provided the original author and source are credited.

\begin{abstract}
This article reviews ethical issues related to genome editing using CRISPR/Cas9 system. The use of CRISPR/ cas9 revives many previous social and ethical issues with humans, other organisms and the environment, such as taking into account the non-maleficence principle in risk assessment, genome editing in germline, safety issues to avoid ecological impairment or the possible use of the technique for genetic enhancement. The new issue is the relatively simple construction and low cost of CRISPR/Cas9 for genome editing, with the possibility of multiple purposes. A public dialogue over the social, ethical and legal implications with the regulatory needs of the system is necessary.
\end{abstract}

Keywords: Genome editing; CRISPR/Cas9 system; Social implications; Legal implications; Ethical implications

\section{Introduction}

For many years molecular biologists have been looking for using cellular repair processes to intervene and modify DNA of organisms by guided genome editing; thus is, to change the organism genome by introducing a new function or correcting a mutation. In the last years CRISPR/Cas9 has been chosen as preferred method for genome editing due to its high degree of fidelity, relatively simple construction and low cost. These characteristics make this technique attractive to be used by any molecular biology lab, but the problem is that can be used for any purpose unless be regulated. This paper reflects on the ethical issues arising from the use of this technique.

\section{Function and Origin of the Crispr/Cas9 System}

The CRISPR/Cas9 system exists in nature as a prokaryotic immune system that confers resistance to foreign genetic elements such as plasmids and bacterial viruses [1,2]. CRISPR refers to "clustered regularly-interspaced short palindromic repeats" consisting in short repetitions of DNA sequences followed by short segments of spacer DNA originated as result of exposition to a bacterial virus or plasmid. The cas (CRISPR-associated) genes code for nuclease or helicase proteins associated to CRISPR repeats with the function of cutting or unwind DNA [3]. CRISPR system functions storing DNA sequences from invaded viruses or plasmid and when the same type of virus invades again, the system recognizes it using the transcribed RNA sequences and directs a cas nuclease to cut the DNA. Cas9 was isolated from bacterium Streptococus pyogenes and is a nuclease able to cut DNA in two active cutting sites at each strand of the double helix of the DNA. Doudna and Charpentier discover that bacteria respond to invading phages by transcribing spacers and palindromic DNA into a long RNA molecule which is cut into pieces (called crRNAs) by using trans-activating RNA (tracrRNA) and protein Cas9 [4]. Later it was discover that the combination of tracrRNA and spacer RNA into a single guideRNA mixed with Cas9 could be programmed to find and cut specific target DNA segments, thus provided with the ability of gene editing [5,6]; crRNA contains a segment able to bind tracrRNA forming a hairpin loop; cas9 is able to modify DNA utilizing crRNA as guide being able to recognize a specific site in host DNA and cleave one or both strands of DNA. A DNA repair template is used for repair process in either Non-Homologous End Joining (NHEJ) or Homology Directed Repair (HDR) inserting a specific DNA sequence for the desire function. Generally a plasmid is used to transfect target cells with CRIPSPR/cas9 or a virus is used as vector. The crRNA is designed in order that Cas9 is able to bind the DNA of the cell only where editing is desired. The DNA repair template is also designed in order to overlap with the sequences on either side of the cut and code for the insertion sequence.

\section{Applications of the Crispr/Cas9 System}

The CRISPR/Cas9 system has many potential applications due to its ability to cut the DNA of any genome at any desired location by introducing the cas9 protein and appropriate guide DNA into a cell $[7,8]$. The system has the ability for genome editing and gene regulation in many types of organisms facilitating the function elucidation of target genes in biology and diseases. Efficient genome editing has been demonstrated in multiple organisms, including bacteria, plants, insects, fish, reptiles, birds and mammals [9-12]. Feng Zhang in 2013 achieved for the first time the genome editing intervention of human cells by engineering a novel version of CRISPR/ Cas9 [13].

CRISPR/Cas9 system can be engineered for many desired functions [14]. The system can introduce DNA in the germline of any organism, and modify somatic genes by genomic editing. CRISPR/Cas9 has been modified to program specific transcription factors to target and activate or silence specific genes [15]. A CRISPR interference platform has been developed for gene silencing, regulating gene expression by binding to the non-template DNA strand of the coding region blocking the transcription elongation process [15]. CRISPR/Cas9 can also inactivate genes inhibiting their transcription by methylation of targeted DNA [16].

CRISPR/Cas9 can be used to create animal models for research to mimic diseases or study development by mutating or silencing genes. For example, a mouse model have been developed to test the deleterious effects of mutations in cancer by introducing loss of 
function mutations in tumor suppressor genes or gaining of function in proto-oncogenes [13]. The animals can be created at the germline level, changing DNA at specific targeted level or anywhere in the genome [17]. Cellular models of human diseases have been created using CRISPR/Cas9 [18].

CRISPR/Cas9 has been also used efficiently to induce genomic alterations in plants improving crop quality or introducing disease resistance [19-23].

The phenomenon of pushing a genetic trait through a population in a non-Mendelian fashion is called gene drive. RNA-guided gene drives based on CRISPR/Cas9 technique may spread altered traits for many generations in a population copying themselves many times in the genome. A method called the mutagenic chain reaction based on CRISPR/Cas9 technique can generate autocatalytic mutations to produce homozygous loss of function mutations, spreading from chromosome of origin to homologous chromosome in the vast majority of somatic and germline cells [24]. Among other uses, gene drives could be used to control damaging invasive species, reverse pesticide and herbicide resistance in insects and weeds in agriculture or prevent the spread of disease [25]. Using this possibility, researchers were able in the lab to spread genes in mosquitoes that prevented them from harboring malaria parasites and also to render female mosquitoes infertile [26].

Genome editing has the potential to cure diseases by disrupting endogenous disease-causing genes, correcting disease causing mutations or inserting new genes with protective functions. The technique can be used in biomedicine to develop tissue-based treatments for cancer and other diseases [27]. For example, CRISPR/ Cas9 may target HIV provirus to mediate excision of the integrated viral genome or prevent cellular entry of the virus [28]. The technique has also shown that can correct the mutation causing cystic fibrosis in intestinal stem cells derived from patients [29].

Also it will facilitate transplanting animal organs into people by eliminating copies of retrovirus present in animal genomes that may harm human recipients [27]. CRISPR/Cas 9 in combination with induced pluripotent stem cells may have the potential to enable the creation of human organs in chimeric pigs, with the possibility of having an unlimited supply of organs not rejected by the immune system of human recipient [30]. In conjunction with blastocyst complementation, the technique CRISPR/Cas9 may produce multigene mutations of essential regulators of vascular and lymphatic tissues in the desired organ, making it ready for transplantation.

Altering DNA in human embryos is also possible. Some scientist from China in 2015 published a paper in the journal Protein \& Cell (previously rejected in Science and Nature due to ethical concerns) reporting the alteration of DNA using abnormal human embryos previously rejected for in vitro fertilization use. They have limited success in correcting a mutation that causes the disease of beta thalassemia using CRISPR/Cas9 [31]. The experiments resulted in changing only some of the genes, and had off-target effects on other genes. Authors stated that the technique is not ready for clinical use. The application of this technique into the zygote or early stage embryo allows the modification of all cells of the organism, including the germline that can be passed to subsequent generations. Scientists are looking for eliminating disease causing genes from the population.

\section{Ethical Issues}

\section{Balance of risks and benefits}

An important ethical issue in research is that benefits must be greater than risks. Greater attention must be place on risks, since they may damage living beings or the environment. The application of CRISPR/Cas9 technique involves risks since it may produce off target mutations, which can be deleterious. A high frequency of off target effects has been found in human cells, but low in mice and zebrafish $[32,33]$. One problem is that large genomes may contain multiple DNA sequences identical or highly homologous to intended target DNA sequence. CRISPR/Cas9 may cleave also these unintended sequences causing mutations which may cause cell death or transformation [34,35]. Efforts have been made to reduce off target mutations, but further improvement is needed, especially for precise modifications needed for therapeutic interventions $[10,12,36]$. Another important problem is the efficient safe delivery of CRISPR-Cas9 into cell types or tissues that are hard to transfect and/or infect.

\section{Ecological disequilibrium}

In experiments using RNA-guided gene drives based on CRISPR/ Cas9 technique, it is necessary to probe specificity considering off target effects. Since gene drive is still operating in created beings, the possibility of mutations off target continues and may increase each generation. If there is risk of transferring genes to other species, then there is risk of transferring modified sequences, passing the negative trait to related organisms even across political borders [25]. The dispersion of gene drive trait may be difficult to control. Furthermore, the disappearance of a whole population targeted by gene drive may carry drastic consequences in the ecosystem equilibrium. For example, other plagues may be developed. Some scientists have warned about the risks of accidental release in the environment of experimental organisms modified using gene drives [37]. This demands careful assessment of each potential application and the need of regulatory norms [37]. Safety measures are necessary in order to avoid disseminations of organisms that may cause ecological damage or affect human health.

\section{Regulations for consumers}

The efficacy of CRISPR/Cas9 technique to obtain precise genetic modifications makes more difficult to identify a genetically modified organism once outside the lab and also to regulate this organisms in the market. Regulatory Agencies such as the Food and Drug Administration in the US, must approve any genetically modified organism for consumers, but it is not clear how they will handle the possibility of an expanded market using CRISPR/Cas9 [38].

Another issue is the regulation of patenting. There are many economic interests involved. For many years transgenic organisms have been patented when they have an industrial use; also human gene sequences have been patented for clinical use; what has made possible an enormous growth of biotechnology. However, the practice of patenting may originate litigations. Already, there have been controversy and frictions among biotechnological companies over patenting CRISPR/Cas9 for therapeutic use in humans. Researchers Zhang versus Doudna and Charpentier disputed over the patent of the technique for use in human cells. Patenting can also be too broad in its applications. On one hand patenting raises the ethical issue that puts the emphasis in profit giving too much power to biotechnological 
companies, but in the other hand patenting may help regulate the field. But the emphasis must be in to commercialize or release only safe products.

\section{Application of CRISPR/Cas9 technique to human germline}

Ethical concerns have been raised regarding the possibility of genome editing in human germline, thus is the genome that can be transmitted to following generations, be from gametes, a fertilized egg or from first embryo divisions [39,40]. Until now, all therapeutic interventions in humans using genome editing has been performed in somatic cells, but the experiment of Chinese researchers Liang and collaborators has created concern over the possibility of making changes in human germline [31]. The difference lies in that intended therapeutic genetic modifications in the germline may be transmitted to following generations. In general, therapeutic genome editing interventions in somatic cells is ethically accepted, considering the balance between risks and benefits and the use of informed consent. But germline cells are not the same, since the CRISPR/Cas9 technique can produce mutations and side effects, unpredictable changes may be transmitted to future generations. Also, there are problems in how to implement informed consent when there are risks and the effects could be transmitted to several generations [41,42]. In light of the possibility to alter human genes, some scientists are calling to a moratorium on applying CRISPR/Cas9 to the human germline. These scientists support basic research on CRISPR in cell lines or in somatic cells, but do not see CRISPR as developed enough for any clinical use in making inheritable changes to humans [43].

Already in 1997 UNESCO issued the Universal Declaration on the Human Genome and Human Rights recommending a moratorium for intervening genetically human germline. In December 2015, the International Summit on Human Gene Editing, which gather members of national scientific academies of America, Britain and China, discussed the ethics of germline modification. They agreed to proceed further with basic and clinical research under appropriate legal and ethical guidelines, but altering of gametocytes and embryos to generate inheritable changes in humans was claimed irresponsible. In addition, they agreed to initiate an international forum where these concerns will be continuously addressed, and regulations in research harmonized across countries [44]. The US National Institute of Health issued a statement, calling for a moratorium, banning NIH-funded research into genomic editing of human embryos [45].

However, in February 2016, British scientists were given permission by regulators to genetically modify human embryos by using CRISPR/ Cas9 and related techniques only for research [46].

Therefore, the genomic editing of human embryos for therapeutic reasons is being hold so far. The risks of hereditable unpredictable genetic mutations are greater than the possible benefits of therapy, affecting the principle of non-maleficence. The technique should be fully safe in order to try therapy in the germline. Furthermore, if damage were introduced, there will be a problem to whom make liable for the damage for following generations.

Once genome editing reaches enough safety level to allow clinical applications for preventing the development of genetic diseases, further discussion will be needed, considering social, legal and ethical implications and the need of regulatory norms to avoid abuses of germline genome editing.

\section{Genome editing for enhancement}

Another ethical issue to discuss is the possibility of non-therapeutic interventions using genome editing. Its use in germ line is ban for safety reasons. But, the efficiency of CRISPR/Cas9 technique increases the possibility to intervene somatic cells in order to match genetics to our life interests. Many phenotypic characteristics have a genetic component, apart from environment, which could be intervened. For example, the technique could be used to enhance performance of athletes or to prevent violent behaviour or diminish addiction. Generally, gene therapy looks to improve the health of a patient for its own benefit, but it may happens in the future that the criminal justice system mandate genome editing of genes related to violence for repeat offenders or violent dangerous criminals [47]. If the intervention is done during development, there are problems of informed consent with minors, since it is questionable that parents or guardians should be allow to decide for them their future for non-health reasons. Socially, there will be a problem if some populations or individuals may be enhanced genetically having advantage over others, for example in intellectual capacity.

\section{Formation of animal chimeras for organ transplantation}

The development of human/animal chimeras for organ transplantation may provide hope for many that have to wait invaluable time for a human organ donor available. But the formation of these chimeras may carry human neural and germ cells [48]. Chimeras have raised ethical concerns over their risk and on the violation of the order of nature, producing moral confusion on how to treat the organism, as animal or human? [30,49,50]. For some, chimeric embryos possess the potential to develop organisms with human-derived cells or tissue, which may affect the identity of the human species, affecting its dignity. But, that an organism contains human cells does not convert the organism into human, neither affects its dignity. The human like characteristic associated to the chimera are only of biological nature, does not affect the moral status of the animal, they do not achieve consciousness for example.

\section{Conclusion}

Many have urged a public dialogue over the social, ethical and legal implications of using genome-editing technique in human germline, but there are other relevant issues to be discussed in relation with this technique as well. The use of CRISPR/cas9 revives many other social and ethical issues, not only with humans, also with other organisms and the environment, such as taking into account the non-maleficence principle in risk assessment, safety issues to avoid ecological impairment or the possible use of the technique for genetic enhancement.

\section{References}

1. Barrangou R, Fremaux C, Deveau H, Richards M, Boyaval P, et al. (2007) CRISPR provides acquired resistance against viruses in prokaryotes. Science 315: 1709-1712.

2. Marraffini LA, Sontheimer EJ (2008) CRISPR interference limits horizontal gene transfer in staphylococci by targeting DNA. Science 322: 1843-1845.

3. Jansen R, Embden JD, Gaastra W, Schouls LM (2002) Identification of genes that are associated with DNA repeats in prokaryotes. Mol Microbiol 43: 1565-1575.

4. Doudna JA, Charpentier E (2014) Genome editing. The new frontier of genome engineering with CRISPR-Cas9. Science 346: 1258096. 
5. Jinek M, Chylinski K, Fonfara I, Hauer M, Doudna JA, et al. (2012) A programmable dual-RNA-guided DNA endonuclease in adaptive bacterial immunity. Science 337: 816-821.

6. Hsu PD, Scott DA, Weinstein JA, Ran FA, Konermann S, et al. (2013) DNA targeting specificity of RNA-guided Cas9 nucleases. Nat Biotechnol 31: 827-832.

7. Zhu LJ (2015) Overview of guide RNA design tools for CRISPR/Cas9 genome editing technology. Front Biol 10: 289-296.

8. Hendel A, Bak RO, Clark JT, Kennedy AB, Ryan DE, et al. (2015) "Chemically modified guide RNAs enhance CRISPR-Cas genome editing in human primary cells". Nature Biotechnology 33: 985-989.

9. Chen L, Tang L, Xiang H, Jin L, Li Q, et al. (2014) Advances in genome editing technology and its promising application in evolutionary and ecological studies. Gigascience 3: 24.

10. Cong L, Ran FA, Cox D, Lin S, Barretto R, et al. (2013) Multiplex genome engineering using CRISPR/Cas systems. Science 339: 819-823.

11. Mali P, Esvelt KM, Church GM (2013) Cas9 as a versatile tool for engineering biology. Nat Methods 10: 957-963.

12. Ma Y, Zhang L, Huang X (2014) Genome modification by CRISPR/Cas9. FEBS J 281: 5186-5193.

13. Chin A (2015) CRISPR/Cas9 Therapeutics: A Technology Overview. Oxford Biotechnolgy.

14. Hale CR, Majumdar S, Elmore J, Pfister N, Compton M, et al. (2012) Essential features and rational design of CRISPR RNAs that function with the Cas RAMP module complex to cleave RNAs. Mol Cell 45: 292-302.

15. Larson MH, Gilbert LA, Wang X, Lim WA, Weissman JS, et al. (2013) CRISPR interference (CRISPRi) for sequence-specific control of gene expression. Nat Protoc 8: 2180-2196.

16. Shalem O, Sanjana NE, Hartenian E, Shi X, Scott DA, et al. (2014) Genome-scale CRISPR-Cas9 knockout screening in human cells. Science 343: 84-87.

17. Maggio I, Gonçalves MA (2015) Genome editing at the crossroads of delivery, specificity, and fidelity. Trends Biotechnol 33: 280-291.

18. Freedman BS, Brooks CR, Lam AQ, Fu H, Morizane R, et al. (2015) "Modelling kidney disease with CRISPR-mutant kidney organoids derived from human pluripotent epiblast spheroids". Nature Communications 6: 8715.

19. Xie K, Yang Y (2013) RNA-guided genome editing in plants using a CRISPR-Cas system. Mol Plant 6: 1975-1983.

20. Shan Q, Wang Y, Li J, Zhang Y, Chen K, et al. (2013) Targeted genome modification of crop plants using a CRISPR-Cas system. Nat Biotechnol 31: 686-688.

21. Feng Z, Zhang B, Ding W, Liu X, Yang DL, et al. (2013) Efficient genome editing in plants using a CRISPR/Cas system. Cell Res 23: 1229-1232.

22. Mao Y, Zhang H, Xu N, Zhang B, Gou F, et al. (2013) Application of the CRISPR-Cas system for efficient genome engineering in plants. Mol Plant 6: 2008-2011.

23. Schaeffer SM, Nakata PA (2015) CRISPR/Cas9-mediated genome editing and gene replacement in plants: Transitioning from lab to field. Plant Sci 240: 130-142.

24. Gantz VM, Bier E (2015) The mutagenic chain reaction: A method for converting heterozygous to homozygous mutations. Science 348:442-444.

25. Esvelt KM, Smidler AL, Catteruccia F, Church GM (2014) Concerning RNA-guided gene drives for the alteration of wild populations. Elife .

26. Science News Staff (2015) "And Science's Breakthrough of the Year is... ".

27. Rath D, Amlinger L, Rath A, Lundgren M4 (2015) The CRISPR-Cas immune system: biology, mechanisms and applications. Biochimie 117: 119-128.
28. Saayman S, Ali SA, Morris KV, Weinberg MS (2015) The therapeutic application of CRISPR/Cas9 technologies for HIV. Expert Opin Biol Ther 15: $819-830$.

29. Schwank G, Koo BK, Sasselli V, Dekkers JF, Heo I, et al. (2013) Functional repair of CFTR by CRISPR/Cas9 in intestinal stem cell organoids of cystic fibrosis patients. Cell Stem Cell 13: 653-658.

30. Feng W, Dai Y, Mou L, Cooper DK, Shi D, et al. (2015) The potential of the combination of CRISPR/Cas9 and pluripotent stem cells to provide human organs from chimaeric pigs. Int J Mol Sci 16: 6545-6556.

31. Liang P, Xu Y, Zhang X, Ding C, Huang R, et al. (2015) CRISPR/Cas9mediated gene editing in human tripronuclear zygotes. Protein Cell 6: 363-372.

32. Hwang WY, Fu Y, Reyon D, Maeder ML, Tsai SQ, et al. (2013) Efficient genome editing in zebrafish using a CRISPR-Cas system. Nat Biotechnol 31: 227-229.

33. Yang H, Wang H, Shivalila CS, Cheng AW, Shi L, et al. (2013) One-step generation of mice carrying reporter and conditional alleles by CRISPR/ Cas-mediated genome engineering. Cell 154: 1370-1379.

34. Fu Y, Foden JA, Khayter C, Maeder ML, Reyon D, et al. (2013) Highfrequency off-target mutagenesis induced by CRISPR-Cas nucleases in human cells. Nat Biotechnol 31: 822-826.

35. Zhang XH, Tee LY, Wang XG, Huang QS, Yang SH, et al. (2015) Off-target Effects in CRISPR/Cas9-mediated Genome Engineering. Mol Ther Nucleic Acids 4: e264.

36. Hsu PD, Scott DA, Weinstein JA, Ran FA, Konermann S, et al. (2013) DNA targeting specificity of RNA-guided Cas9 nucleases. Nat Biotechnol 31: 827-832.

37. Oye KA, Esvelt K, Appleton E, Catteruccia F, Church G, et al. (2014) Biotechnology. Regulating gene drives. Science 345: 626-628.

38. Ledford H (2015) CRISPR, the disruptor. Nature 522: 20-24.

39. Otieno MO (2015) CRISPR/Cas9 Human Genome Editing: Challenges, Ethical Concerns and Implications. J Clin Res Bioeth 6: 253.

40. Ishii $\mathrm{T}$ (2015) Germline genome-editing research and its socioethical implications. Trends Mol Med 21: 473-481.

41. Billings PR, Hubbard R, Newman SA (1999) Human germline gene modification: a dissent. Lancet 353: 1873-1875.

42. Frankel MS, Chapman AR (2000) American Association for the Advancement of Sciences. Human Inheritable Genetic Modifications. Assessing Scientific, Ethical, Religious and Policy Issues, pp 1-82.

43. Lanphier E, Urnov F, Haecker SE, Werner M, Smolenski J (2015) Don't edit the human germ line. Nature 519: 410-411.

44. Steven O, Editor, Committee on Science, Technology, and Law; Policy and Global Affairs; National Academies of Sciences, Engineering, and Medicine (2015) International Summit on Gene-Editing.

45. Collins F (2015) Statement on NIH funding of research using geneediting technologies in human embryos. The NIH Director - National Institutes of Health (NIH).

46. Gallagher J (2016) "Scientists get 'gene editing' go-ahead". BBC News (BBC).

47. Cheng M (2016) "Britain approves controversial gene-editing experiments". AP News.

48. Polcz S, Lewis A (2016) CRISPR/Cas9 and the Non-Germline NonControversy.

49. Hermerén G (2015) Ethical considerations in chimera research. Development 142: 3-5.

50. Hyun I (2015) From naïve pluripotency to chimeras: a new ethical challenge? Development 142: 6-8. 